\title{
Entrando en el mundo nano
}

\author{
Alfred NordmanN*
}

H

ay mucho de cierto en el entusiasta slogan que alguna vez utilizó la

Asociación Internacional de Nanotecnología de San Francisco: “... es un mundo nano, hagámoslo un mejor lugar". El slogan expresa los términos en los que somos invitados a contribuir en el desarrollo responsable de la nanotecnología: el mundo nano ya está con nosotros; no puede ser cuestionado o rechazado. Si entramos al mundo nano animados y con buen entusiasmo, seremos más que bienvenidos a la hora de colaborar en decorarlo y mejorarlo.

En lugar de entrar a ese mundo tan optimistas, debemos, por el contrario, intentar dar un paso atrás y desafiar esta invitación. Y es que el problema comienza con la definición de nanotecnología. Describir y definir la nanotecnología no es una tarea inocente. Dependiendo de cómo la definamos, el resultado puede ser cualquier cosa, desde el asombro paralizante o la especulación impotente hasta la concienciación crítica o la modelización de la política, en el otro extremo.

Nuestra definición nos conducirá a asumir la expectativa de que todos los aspectos de nuestras vidas serán revolucionados, o de lo contrario, a creer que estamos ante una tendencia de moda, que, sin embargo, está lejos de ser de poca importancia.

La manera más modesta de definir el término, dígase el establecimiento del más bajo común denominador sobre lo que se entiende por investigación nanotecnológica, no es aún, y bajo ningún motivo, inocente. Y es que el más bajo común denominador sigue siendo demasiado grande para poder hacer una reflexión significativa o tomar acciones políticas. Definiciones limitadas en términos del tamaño o propiedades novedosas sirven meramente para establecer el dominio de la investigación nanocientífica. La nanotecnología, entonces, aparece como potencial casi infinito de posibles aplicaciones que surgen de trabajar con esos objetos de investigación.

Por consiguiente, una típica afirmación es que la nanotecnología hace uso de las propiedades que ocurren sólo en la escala molecular de 1 a 100 nanómetros $\left(10^{-9}-10^{-7}\right.$ metros $)$ y que son distintas a las propiedades macroscópicas. El oro es usualmente utilizado como un ejemplo de ello: su

* Universidad Técnica de Darmstadt (Alemania).www.nanoOffice.eu 
color, su inercia química y, por tanto, sus impactos en la salud son todos bien conocidos. Sin embargo, si su composición química se mantiene y el oro se reduce meramente al tamaño de una nanopartícula, tales propiedades cambian. Aquí es donde la nanotecnología entra en juego. Abarca todo lo que hace uso de ese tipo de cambios.

Y ¿cuáles podrían ser éstos? En este punto, la definición ya no ofrece ninguna guía y más bien nos deja con posibilidades ilimitadas. Si los nanotubos de carbono poseen propiedades ópticas interesantes, ¿no sería concebible construir un tipo de computadora completamente diferente, una que ya no trabaje con base en la electrónica binaria sino más bien con base en la fotónica, es decir, usando el espectro del color? Si las fibras nanoestructuradas son especialmente ligeras y fuertes, ¿no sería posible hacer una cuerda con ellas para unirlas a un elevador que vaya al espacio? Si los procesos de autoorganización o autoensamblaje de la naturaleza están entre las nuevas propiedades, ¿no podríamos imaginarnos nanosistemas o, incluso, pequeños robots que produzcan y se reproduzcan a sí mismos? Y así sucesivamente.

Si así definimos la nanotecnología, comenzamos a creer que es capaz de cualquier cosa y terminamos con una determinación del futuro -sea éste cercano, o no- en el que nada es como solía ser previamente. De este modo, la nanotecnología elude el poder de nuestra imaginación y se convierte en algo que no está disponible al pensamiento crítico o la acción política. Es una marioneta en un juego de vagas suposiciones.

Sin embargo, la "nanotecnología" puede ser definida en una forma totalmente diferente; como un concepto político que vincula trayectorias de investigación heterogéneas. Dichas trayectorias tienen, desde luego, algo en común. Se espera que todas ellas abran nuevos mercados y todas ellas manipulan propiedades o estructuras en el rango de 1 a 100 nanómetros. Pero nótese que aquí, no son esos factores en común la clave sino más bien los programas específicos que confluyen en este punto. Así, en vez de hablar sobre nanotecnología, deberíamos en cambio estar hablando sólo acerca de diferentes nanotecnologías.

¿Significa, entonces, que no hay nada que decir sobre la nanotecnología en general? Bueno, si lo hacemos, no estamos hablando acerca de programas de investigación y trayectorias tecnológicas. Nanotecnología en lo singular es sólo una palabra relativa a lo que la gente espera hoy de la tecnología -una conquista del espacio en la que se supone que las soluciones para todos nuestros problemas yacen ahí ocultas. De hecho, y a diferencia de la guerra contra el cáncer, la carrera armamentista o el proyecto genoma humano, la investigación a nanoescala no es impulsada por una preocupación en asuntos específicos, sino más bien, está orientada hacia un espacio 
de posibilidades. La nanotecnología no está interesada en representaciones científicas de la naturaleza ni en dispositivos técnicos que funcionan. En cambio, la nanociencia es un intento explicatorio para reclamar territorio extranjero y habitar un nuevo mundo o, más bien, una región inexplorada del mundo. El éxito de los investigadores siempre es un tipo de realización técnica, dígase la habilidad de actuar a nanoescala. Aunque esto no altera fundamentalmente nuestra comprensión básica de la naturaleza, es intelectualmente estimulante y finalmente "experimentar" las condiciones reales a nanoescala, cara a cara y con las manos sobre la materia. Por tanto, como con el programa espacial, el éxito científico y tecnológico a nanoescala consiste en la habilidad de ver, de moverse, de mover cosas y de actuar a esa escala, esto es, en la adquisición de capacidades que permitan habitar el espacio interior de modo similar a cómo hemos conquistado el mundo salvaje o cómo hemos empezado a reclamar el espacio exterior.

Entrando en el mundo nano y reconociendo el reto de colonizar y organizar ese espacio interior, vale preguntarse entonces cuáles son algunos de los retos de diseño que enfrentan los políticos, los ciudadanos y los nanotecnólogos. Propongo considerar, 1) el diseño de interfaces, y 2) el diseño de la nave espacial terrestre.

\section{EL DISEÑO DE INTERFACES}

Desde la perspectiva de muchos investigadores y comentaristas, la nanotecnología está generando un curioso cambio en la concepción tradicional de la tecnología. La visión tradicional sugiere lo siguiente: la naturaleza solía ser un lugar extraño y mágico con algo de espiritualidad en cada árbol. A falta de previsibilidad racional y control, no hay nada que podemos hacer más que rezar a esas cosas. La tecnología nos liberó de tal predicamento dándonos control a la par que desencanto y alienación. Sin embargo, ahora, las visiones tecnológicas más avanzadas en computación, genética o nanotecnología llegan a un límite en el que la tecnología se convierte en algo mágico, regresándonos a nuestro punto de partida, es decir, a un encantado y extraño estado de la naturaleza que, en realidad, en un principio, ya habíamos considerado insostenible cuando pensábamos en controlarla, computarla e incluso manipularla y dominarla. Este tipo de "tecnología naturalizada" es regresiva en tanto que nos devuelve a un estado de ignorancia de cara a nuestras invenciones técnicas. Los productos de nuestras mentes y acciones se confrontan; tal vez dejándonos como parte de esa naturaleza incomprendida.

La noción de diseño de interfaces hace un llamado a los ingenieros en el sentido de reflexionar acerca del propósito de la tecnología y sobre la 
necesidad de combatir su regresión. Por ejemplo, si uno fuera a diseñar un dispositivo nanoescalar que puede desplazarse, afectar cosas y replicarse autónomamente, uno también tendría que aprender a rastrearlo, monitorearlo, avistarlo y controlarlo. Para la tecnología naturalizada necesitaremos descubrir tecnologías de confinación que permitan atarla a la escala de la acción humana. Tales tecnologías de confinación implican el diseño de interfaces, la determinación política en cuanto a especificaciones de diseño, inclusive técnicas conceptuales o literarias de conceptos venideros, así como la socialización de la tecnología naturalizada.

\section{El Diseño de la Tierra como una Nave Espacial}

Cuando Richard Feynman publicó su discurso de 1959: "There is plenty of room at the bottom", no estaba, en realidad, fundando la nanotecnología, pero sí extendió una "invitación a entrar a un nuevo campo de la física". Mientras Feynman articuló su visión de espacio interno sin fronteras, complementó la obsesión de sus días por la exploración del espacio exterior. Como lo ha señalado Astrid Schwarz (2005), "hay suficiente espacio ahí abajo", significa que podríamos estar viviendo en un mundo limitado de recursos escasos, pero que hay una abundancia global ilimitada en el mundo nano -por tanto, colonicemos el mundo tal y como los contemporáneos de Feynman soñaron en escapar de la limitada condición humana por medio de la colonización de Marte. Dicha actitud es raramente enunciada explícitamente, pero es implícita en la creencia de un todopoderoso arreglo nanotecnológico (no hay problema que la nanotecnología no vaya a resolver) y en la ausencia de una reflexión sobre la eventual aparición de límites en el conocimiento y en la capacidad de control, ello, sobre todo, de cara a la complejidad que caracteriza la nanoescala.

Ahora bien, en lugar de apostarle, al futuro, una ruta alternativa es comenzar con los problemas medioambientales inmediatos y dirigir específicamente los programas de investigación en nanotecnología a la solución de tales problemas. Con el fin de alcanzar esta última ruta, será necesario entonces priorizar las promesas medioambientales de la nanotecnología en cuanto a su urgencia y relevancia social. La elección básica está, pues, entre el diseño consciente, la salvaje y peligrosa "Tierra como nave espacial" y la mera espera de los supuestos beneficios ambientales que se presumen de la mano de capacidades ilimitadas y de una recién descubierta abundancia.

En el recuento ofrecido hasta ahora, los nanoingenieros y nanoéticos trabajan en el mundo nano buscando caminos para acomodar a los seres humanos en éste. A la apertura inicial del mundo nano (hay mucho espacio 
y, por tanto, un supuesto ilimitado potencial tecnológico) le corresponde entonces una aparente apertura del espacio social. Considerando lo anterior, la investigación en nanoescala y otras tecnologías "facilitadoras", ${ }^{1}$ parecen permitir posibilidades únicas para la modelización social y el establecimiento de la agenda pública. De hecho, la transformación de la "nanotecnología", en determinadas nanotecnologías, debería y podría surgir de procesos democráticos. Pero, si el desarrollo de las nanotecnologías queda mejor enmarcado en el discurso de la globalización o en el de la colonización, es importante reconocer que ese espacio social no es, bajo ningún motivo, un espacio vacío donde todo es posible. Y es que cuando los ciudadanos del mundo se reúnan para reflexionar sobre los programas nanotecnológicos y sus promesas, seguramente encontrarán que su espacio discursivo, aparentemente abierto, está ocupado en buena medida por nociones de eficiencia, sustentabilidad, innovación, competitividad, supervivencia económica, entre otras cuestiones. En consecuencia, la labor de ingenieros y especialistas en ética no es sólo dilucidar visiones nanotecnológicas de modo específico, sino también resistir a los intentos de cierre prematuro. Necesitan desenmarañar demandas incuestionables referentes a las necesidades de innovación, la competencia global, las predicciones de mercado, los supuestos beneficios sociales o las necesidades humanas que han de ser asumidas.

Un poco menos entusiasta y con al menos una pizca de resignación, deberíamos modificar, por lo menos ligeramente, el eslogan que abre la presente reflexión: "Es un mundo nano - hagamos lo mejor de éste".

Para una revisión extensa de los puntos aquí tratados, léase: Nordmann, 2007.

\section{Bibliografía}

Nordmann, Alfred (2007). 'Design Choices in the Nanoworld: A Space Odyssey' en Marian Deblonde, Lieve Goorden et al. "Nano Researchers Facing Choices." Christiane Timmerman y Barbara Segaert (eds). The Dialogue Series. Universitair Centrum Sint-Ignatius Antwerpen. Vol. 10: 13-30.

Schwarz, Astrid (2005) "Shrinking the Ecological Footprint with NanoTechnoScience?" en Davis Baird, Alfred Nordmann, Joachim Schummer (eds.) Discovering the Nanoscale, Amsterdam: ios Press, pp. 203-208.

${ }^{1}$ Enabling technologies, en inglés. Se refiere a tecnologías que permiten el avance de la ciencia y la tecnología en su conjunto. Dentro de ésas, la literatura precisa la biotecnología, la electrónica e informática, entre otras. 


\section{EVENTOS}

- 18-20 de febrero de 2009

\section{Nano Tech 2009}

Conference Tower. Tokyo Big Sight. Japón. Evento internacional sobre el avance de la nanotecnología y su presencia en el mercado. Organizada por el Prof. Tomoji Kawai del Instituto de Investigación Científica e Indus-

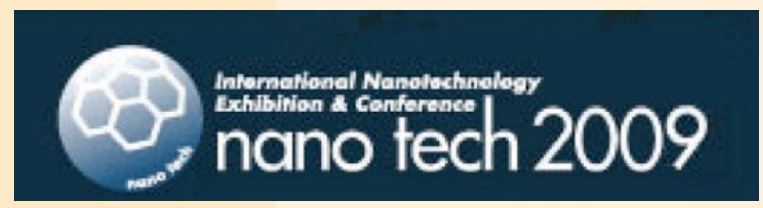
trial de la Universidad de Osaka; el Dr. Eiichi Maruyama, asesor del Centro para Estrategias de la Propiedad Intelectual del RIKEN; entre otros miembros de la Universidad de Tohoku, el Instituto de Investigación de Mitsubishi, el Instituto Nacional para el Avance de la Ciencia y la Tecnología (AIST), etcétera.

Más información en: http://www.nanotechexpo.jp/en/index.html

\section{Nanotoxicology: Health \& Environmental Impacts}

\section{International Conference on the Environmental Implications and Applications of Nanotechnology}

Amherst, Massachusetts, Estados Unidos. Conferencia internacional organizada por el Environmental Institute y la Environmental Protection Agency de Estados Unidos. Temas a tratar: caracterización, detección y análisis; nanotecnología verde; regulación y asuntos de legislación en nanotecnología; destino ambiental y movimiento de nanomateriales; biocapacidad, toxicidad y grados de exposición de nanomateriales; nanotecnología para el control y remediación ambiental.

Más información en: http://www.umass.edu/tei/conferences/NanoConference/index. html 